\title{
Pasteurella multocida Peritonitis in a 3-Year-Old Patient Undergoing Peritoneal Dialysis: Case Report and Review of the Literature
}

\author{
Hiroshi Tamura*, Shohei Kuraoka, Tomomi Nishi, Yuko Hidaka, Hiroko Nagata, Hitoshi Nakazato \\ Department of Pediatrics, Faculty of Life Sciences, Kumamoto University, Kumamoto, Japan
}

Email address:

bohm1905HT@kuh.kumamoto-u.ac.jp (H. Tamura)

${ }^{*}$ Corresponding author

To cite this article:

Hiroshi Tamura, Shohei Kuraoka, Tomomi Nishi, Yuko Hidaka, Hiroko Nagata, Hitoshi Nakazato. Pasteurella multocida Peritonitis in a 3-Year-Old Patient Undergoing Peritoneal Dialysis: Case Report and Review of the Literature. American Journal of Pediatrics.

Vol. 4, No. 3, 2018, pp. 52-55. doi: 10.11648/j.ajp.20180403.12

Received: June 1, 2018; Accepted: June 26, 2018; Published: July 26, 2018

\begin{abstract}
Pasteurella multocida has been rarely reported to cause peritonitis in children. Peritoneal dialysis (PD) peritonitis is associated with high mortality despite appropriate treatment; therefore, its early detection is essential. Here, we report the case of a 3-year-old girl, undergoing treatment with PD, who developed peritonitis caused by $P$. multocida after a domestic cat scratched a dialysate bag used for PD. The patient was treated with antibiotics and her condition was soon in remission. PD peritonitis can occur despite the isolation of equipment, as in this case, it is preferable to completely avoid having pets if possible. But, there is the benefit of pets which heal the minds of humans with a chronic illness. We propose that it is preferable for PD patients to keep pets away from rooms where they store PD equipment and perform PD.
\end{abstract}

Keywords: Peritonitis, Peritoneal Dialysis, Pasteurella multocida, Pet, Hygiene

\section{Introduction}

Peritonitis is a major serious complication of peritoneal dialysis (PD) therapy and is one of the important reasons for the discontinuation of PD. Pasteurella multocida is a Gram negative zoonotic pathogen found in the oral cavities of domestic cats, dogs, and other wild and domestic animals. Infectious diseases caused by the transmission of $P$. multocida to humans have been reported to result in systemic infections including sepsis and local infections involving soft tissues or respiratory system. To date, 29 cases of PD-associated P. multocida peritonitis linked to animal contact have been reported in the literature (Table 1) [1-29]. Among these, only two cases have been reported in pediatric patients undergoing PD (cases 2 and 3).

We report the case of a 3-year-old patient who experienced PD-associated peritonitis due to $P$. multocida.

\section{Case}

A three-year-old female patient with end-stage renal disease caused by congenital nephrotic syndrome of the Finnish type was treated with automated peritoneal dialysis (APD) and continuous ambulatory peritoneal dialysis (CAPD) for 26 months. Her mother found scratches on the dialysate bag that was used for PD. Two days later, the patient was admitted to our hospital with slight fever and cloudy effluent in the dialysis bag. She was $86.3 \mathrm{~cm}$ tall, and weighed $10.2 \mathrm{~kg}$. Physical examination upon admission revealed no abdominal pain. There were no signs of either tunnel or exit-site infections or damage to the catheter and tube.

The patient's vital signs were as follows: a respiratory rate of 120 beats $/ \mathrm{min}$, a heart rate of 120 beats $/ \mathrm{min}$, blood pressure of $108 / 62 \mathrm{mmHg}$, and a temperature of $37.4^{\circ} \mathrm{C}$. No tenderness was present in her abdomen. Her laboratory data on admission showed a white blood cell (WBC) count of $12,800 / \mu \mathrm{L}$ with $84.9 \%$ neutrophils, blood urea nitrogen of $50.7 \mathrm{mg} / \mathrm{dL}$, creatinine level of $4.55 \mathrm{mg} / \mathrm{dL}$, and C-reactive protein level of $9.73 \mathrm{mg} / \mathrm{dL}$. Analysis of her initial dialysis fluid revealed a cell count of $16 \mathrm{WBC} / \mu \mathrm{L}$.

Gram staining revealed Gram negative bacteria in her peritoneal fluid and $P$. multocida was identified via bacterial culturing. Minimum inhibitory concentrations of known 
antibiotics against the pathogen were piperacillin $<1 \mu \mathrm{g} / \mathrm{ml}$, cefazolin $<0.5 \mu \mathrm{g} / \mathrm{ml}$, ceftriaxone $<0.25 \mu \mathrm{g} / \mathrm{ml}$, imipenem/cilastatin $0.25 \mu \mathrm{g} / \mathrm{ml}$, and levofloxacin $<0.25 \mu \mathrm{g} / \mathrm{ml}$.

APD was changed to CAPD therapy. The patient was treated with a combination of intraperitoneal cefazolin four times a day and intravenous piperacillin once a day.

She developed a high fever, and the WBC count of the fluid dialysate increased to $778 / \mu \mathrm{L}$ on the day following hospitalization. The high fever disappeared on the second day of treatment, and the laboratory findings improved by the 9th day. The treatment was changed to only intravenous cefazolin on the 9th day after starting the combination therapy, and was subsequently changed to oral cefdinir on the 12th day.

The patient had a domestic cat. Her mother had not found the cat playing with, biting, or puncturing the PD tube or bags but found scratches on a bag used for PD. The patient had a history of close contact with her cat. Although she had undergone home PD for 2 years, she had no history of PD-related peritonitis.

\section{Discussion}

Pasteurella is a genus comprising 13 species of Gram negative facultative anaerobic coccobacilli, found in the normal flora of several animals, including cats (up to approximately $90 \%$ of cat populations) and dogs (up to approximately $60 \%$ of $\operatorname{dog}$ populations) [30]. In addition, it has also been reported that one-third of healthy livestock breeds are oropharyngeal carriers of this pathogen. Human infection occurs following animal bites, scratches, or even licks. P. multocida usually causes skin, soft tissue, respiratory tract, bone and joint infections. It is an uncommon cause of peritonitis in patients undergoing peritoneal dialysis. Peritonitis caused by $P$. multocda is estimated to comprise approximately $8 \%$ of PD-associated peritonitis [9]. However, only 30 cases of $P$. multocida peritonitis in association with PD have been reported (Table 1), of which 3 were pediatric (cases 1-3). In most cases, the patients had a domesticated cat, and APD was more commonly used method of dialysis than CAPD. The reason for this remains unclear, but it is postulated that the relatively short duration of PD exchange in CAPD reduces the chances of cats coming into contact with $\mathrm{PD}$ tubes. Further, long PD tubes necessary for APD and the warming dialysate bags used appear to be more attractive toys for cats.

In this case, the pathogen was observed using Gram staining. However, 14 out of the 23 cases examined have been reported to be negative, and the detection rate was low. The reason for this is remaind unclear, but it is likely that $P$. multocida is susceptible to heat and ultraviolet rays [9, 27].

Peritonitis caused by P. multocida develops within 24 hours of exposure, and its subjective symptoms are strong [23]. In this case, the onset of peritonitis required 2 days. It is possible that very few pathogens were present as contaminants and because the dialysis methods comprised eight cycles of APD and two cycles of CAPD per day, any bacteria were washed away and their growth was suppressed.

Table 1. Cases of Pasteurella multocida peritonitis reported in the literature.

\begin{tabular}{|c|c|c|c|c|c|c|c|}
\hline case & Age(years) & sex & PD type & animal & leak & Gram staining & reference \\
\hline 1 & 4 & F & APD,CAPD & cat & - & Gram(-) rod & This case \\
\hline 2 & 7 & $\mathrm{~F}$ & NIPD & cat & - & Gram(-) rod & 1 \\
\hline 3 & 12 & $\mathrm{~F}$ & CCPD & cat & + & - & 2 \\
\hline 4 & 16 & M & APD & cat & NR & NR & 3 \\
\hline 5 & 21 & $\mathrm{~F}$ & CCPD & cat & + & NR & 4 \\
\hline 6 & 22 & $\mathrm{~F}$ & CCPD & cat & - & - & 5 \\
\hline 7 & 24 & $\mathrm{~F}$ & CCPD & cat & - & NR & 6 \\
\hline 8 & 25 & $\mathrm{~F}$ & CAPD & cat & - & - & 7 \\
\hline 9 & 25 & M & CCPD & cat & - & - & 8 \\
\hline 10 & 29 & F & NIPD & cat & - & - & 9 \\
\hline 11 & 36 & $\mathrm{~F}$ & CCPD & cat & - & - & 10 \\
\hline 12 & 38 & M & CCPD & cat & + & - & 11 \\
\hline 13 & 42 & F & CCPD & cat,dog & + & - & 12 \\
\hline 14 & 46 & $\mathrm{~F}$ & APD & NR & NR & Gram(-) rod & 13 \\
\hline 15 & 46 & $\mathrm{~F}$ & CCPD & cat & - & Gram(-) rod & 14 \\
\hline 16 & 47 & $\mathrm{~F}$ & CCPD & cat & - & Gram(-) rod & 15 \\
\hline 17 & 48 & $\mathrm{~F}$ & CAPD & cat & NR & - & 16 \\
\hline 18 & 48 & $\mathrm{~F}$ & CAPD & dog & NR & - & 17 \\
\hline 19 & 49 & M & CAPD & cat,dog & - & NR & 18 \\
\hline 20 & 49 & M & NIPD & cat & NR & Gram(-) rod & 19 \\
\hline 21 & 52 & M & CCPD & cat & - & NR & 20 \\
\hline 22 & 54 & M & CCPD & cat & + & Gram(-) rod & 21 \\
\hline 23 & 55 & F & CCPD & cat & + & - & 22 \\
\hline 24 & 55 & $\mathrm{M}$ & CAPD & cat & + & NR & 23 \\
\hline 25 & 55 & M & CCPD & cat & + & - & 24 \\
\hline 26 & 58 & M & CCPD & cat & + & Gram(-) rod & 25 \\
\hline 27 & 58 & M & CCPD & cat & - & - & 26 \\
\hline 28 & 73 & M & CAPD & cat & - & NR & 27 \\
\hline 29 & 73 & F & CAPD & cat & - & Gram(-) rod & 28 \\
\hline 30 & 75 & $\mathrm{M}$ & CAPD & cat & + & - & 29 \\
\hline
\end{tabular}

Abbreviation: M; male, F; female, PD; peritoneal dyalysis, CAPD; continuous ambulatory peritoneal dialysis, CCPD; continuous cyclic peritoneal dialysis, APD; automated peritoneal dialysis, NIPD; nocturnal intermittent peritoneal dialysis, NR; not reported, Gram (-) rod; gram negative rod-shaped bacterium. 
The number of dialysis cases in Japan is approximately 325,000 , only $3 \%$ of which are treated by PD. However, PD comprises approximately $90 \%$ of the total number of dialysis cases in children. The number of PD cases in Japan relative to other regions of the world is currently small but is likely to increase in the future. The ownership rate of dogs and cats in Japan is approximately $16 \%$ with an increasing yearly trend. Pets ownership are increasing in the West [31]. Therefore, PD peritonitis caused by pets appears to be on the rise, and $P$. multocida peritonitis is a significant issue. It is important to inform patients undergoing PD regarding the potential for transmission of diseases from pets, and the importance of maintaining local environmental hygiene.

\section{Conclusion}

PD peritonitis may be fatal. PD peritonitis can occur despite the isolation of equipment, as in this case, it is preferable to completely avoid having pets if possible. But, there is the benefit of pets which heal the minds of humans with a chronic illness. We propose that it is preferable for patients undergoing PD to keep pets away from rooms where they store PD equipment and perform PD.

\section{Conflict of Interest}

All the authors do not have any possible conflicts of interest.

\section{References}

[1] Sol PM, van de Kar NC, Schreuder MF. Cat induced Pasteurella multocida peritonitis in peritoneal dialysis: a case report and review of the literature. Int J Hyg Environ Health. 2013; 216: 211-213.

[2] Loghman-Adham M. Pasteurella multocida peritonitis in a patient undergoing peritoneal dialysis. Pediatr Nephrol. 1997; 11: 353-354.

[3] Chadha V, Warady BA. Capnocytophaga canimorsus peritonitis in a pediatric peritoneal dialysis patient. Pediatr Nephrol. 1999; 13: 646-648.

[4] Malik A, Mailey KS, Bastani B. Pasteurella multocida peritoneal dialysis-associated peritonitis: a report of two cases and review of the literature. J Nephrol. 2005; 18: 791-793.

[5] Van Langenhove G, Daelemans R. Pasteurella multocida as a rare cause of peritonitis in peritonealdialysis. Nephron. 2000; 85: 283-284.

[6] Kanaan N, Gavage P, Janssens M. Pasteurella multocida in peritoneal dialysis: a rare cause of peritonitis associated with exposure to domestic cats. Acta Clin Belg. 2002; 57: 254-256.

[7] Il K, Young WK, Sunglin C. Cat-induced Pasturella multocida peritonitis in continuous ambulatory penitoneal dialysis. Kidney Res Clin Pract. 2004; 33: 65-67.

[8] Elsey RM, Carson RW, DuBose TD Jr. Pasteurella multocida peritonitis in a HIV-positive patient on continuous cycling peritoneal dialysis. Am J Nephrol. 1991; 11: 61-63.

[9] Kazuko I, Yoshihiro T, Tsutomu T, et al. Two case reports of Pasteurella peritonitis in peritoneal dialysis. J. jpn. Soc. Dial. Ther. 2008; 41: 213-218.

[10] Mugambi SM, Ullian ME. Bacteremia, sepsis, and peritonitis with Pasteurella multocida in a peritoneal dialysis patient. Perit Dial Int. 2010; 30: 381-383.

[11] Helbert R, Giraldian J, Trevejo N. Pets or Pest: Peritoneal Dialysis-related Peritonitis due to Pasteullea multocida. J Microbiol Immunol Infect. 2010; 43: 155-158.

[12] Uribarri J, Bottone EJ, London RD. Pasteurella multocida Peritonitis: are peritoneal patients on cyclers at increased risk? Perit Dial Int. 1996; 16: 648-649.

[13] Breton-Jr M, Salavert LM, Viudes FA. Perez B. C., Gobernado S. M. Abdominal infection by Pasteurella spp. A report of 3 cases. Rev Clin Esp. 2000; 200: 139-142.

[14] Olea T, Hevia C, Bajo MA, et al. Pasteurella multocida and Candida albicans peritonitis. Nefrologia. 2006; 26: 136-138.

[15] Musio F, Tiu A. Pasteurella multocida peritonitis in peritoneal dialysis. Clin Nephrol. 1998; 49: 258-261.

[16] Sillery J, Hargreaves J, Marin P, et al. Pasteurella multocida peritonitis: another risk of animal-assisted therapy. Infect Contr Hosp Epidemiol. 2004; 25: 5-6.

[17] Antony SJ, Oglesby KA. Peritonitis associated with Pasteurella multocida in peritoneal dialysis patients-case report and review of the literature. Clin Nephrol. 2007; 68: 52-56.

[18] Al-Fifi YS1, Sathianathan C2, Murray BL, et al. Pets are 'risky' business' for patients undergoing continuous ambulatory peritoneal dialysis. 2013; 24: e96-8.

[19] Hamai K, Imai H, Ohtami H. Repeated cat-associated peritonitis in a patient on automated nocturnal intermittent peritoneal dialysis. Clin Exp Nephrol. 1999; 1:59- 61.

[20] Mat O, Moenens F, Beauwens R, et al. Indolent Pasteurella multocida peritonitis in a CCPD patient. 25 Years of "cat-bite peritonitis": a review. Perit Dial Int. 2005; 25: 88-90.

[21] London RD, Bottone EJ. Pasteurella multocida: zoonotic cause of peritonitis in a patient undergoing peritoneal dialysis. Am J Med J. 1991; 91: 202-204.

[22] Paul RV, Rostand SG. Cat-bite peritonitis: Pasteurella multocida peritonitis following feline contamination of peritoneal dialysis tubing. Am J Kidney Dis.1987; 10: 318-319.

[23] JohJ, Padmanabhan R, Bastani B. Pasteurella multocida peritonitis following cat bite of peritoneal dialysis tubing. Am J Nephrol. 1998; 18: 258-259.

[24] Frankel AH, Cassidy MJ. Pasteurella multocida peritonitis in CAPD: beware of the cat. Perit Dial Int. 1991; 11: 184-185.

[25] Malik A, Al Aly K, Mailey KS. Pasteurella multocida peritoneal dialysis-associated peritonitis. A report of two cases and review of the literature. J Nephrol. 2005; 18: 791-793.

[26] Satomura A, Yanai, Arashima Y, Kumasaka K, et al. Peritonitis associated with Pasteurella multocida: molecular evidence of zoonotic etiology. Ther Apher Dial. 2010; 14: 373-376. 
[27] Mackay K, Brown L, Hudson F. Pasteurella multocida peritonitis in peritoneal dialysis patients: beware of the cat. Perit Dial Int. 1997; 17: 608-610.

[28] Cooke F, Kodjo A, Clutterbuck E. A case of Pasteurella multocida peritoneal dialysis-associated peritonitis and review of the literature. Int J Infect Dis. 2004; 8: 171-174.

[29] Kitching AR, Macdonald A, Hatfield PJ. Pasteurella multocida infection in continuous ambulatory peritoneal dialysis. $\mathrm{N} \mathrm{Z}$ Med. 1996; 59: 109.
[30] Griego RD, Rosen T, Orengo IF, Wolf JE. Dog, cat and human bites: review. J Am Acad Dermatol. 1995; 33: 1019-29.

[31] Philippe GP, Philippe LW, Mauro V, et al. Pasteurella species peritoneal dialysis-associated peritonitis: Household pets as a risk factor. Can J infect Dis Med Microbiol. 2015; 26: 52-55. 